\title{
ANÁLISIS DEL SIGNIFICADO DE LAS OCUPACIONES ATRIBUIDAS A SER MUJER Y MADRE PARA MUJERES CON DISCAPACIDAD INTELECTUAL EN LA CIUDAD DE PUNTA ARENAS
}

\author{
MEANING ANALYSIS OF OCCUPATIONS ATTRIBUTED TO BE A WOMAN AND MOTHER FOR \\ WOMEN WITH INTELLECTUAL DISABILITY IN THE CITY OF PUNTA ARENAS \\ María Dehays P. ${ }^{1}$ Melissa Hichins A. ${ }^{2}$ Vanessa Vidal C. ${ }^{3}$
}

\section{Resumen}

Esta investigación pretende dar a conocer cuáles son los significados que las mujeres con discapacidad intelectual le brindan a las experiencias de ser mujer y madre, junto con los factores que les facilitan y obstaculizan este proceso. Se utilizó una metodología cualitativa desde la mirada del paradigma interpretativo con un enfoque metodológico descriptivo-exploratorio; el tipo de diseño que se utilizó es el interaccionismo simbólico, en base a estudio de caso, donde la muestra está compuesta por tres mujeres con discapacidad intelectual y los resultados se analizaron según la Teoría Fundamentada.

La principal conclusión obtenida en esta investigación es que, los significados atribuidos por este grupo de mujeres estudiadas al ser mujer y madre son la feminidad y tareas domésticas, unidos a factores emocionales y de cuidados básicos. Un hallazgo importante es, que este colectivo de personas es vulnerable a que haya una transgresión de género; al igual que la violación de sus derechos humanos más básicos en su vida diaria. Ellas no ejercen sus ocupaciones significativas de forma independiente, siendo víctimas de una situación de Apartheid Ocupacional e Injusticia Ocupacional.

\section{Palabras clave}

Discapacidad intelectual, Mujer, Significado, Maternidad, Injusticia Ocupacional, Derechos Humanos.

\footnotetext{
${ }^{1}$ Licenciada en Ciencia de la Ocupación, Universidad de Magallanes, 65942471Constanza.dehays@gmail.com

${ }^{2}$ Licenciada en Ciencia de la Ocupación, UMAG, 66414621 Melissa.hichis@gmail.com

${ }^{3}$ Licenciada en Ciencia de la Ocupación, UMAG, 71487774
} 


\begin{abstract}
This research aims to show what are the meanings that women with intellectual disabilities give to the experiences of being a woman and mother, alongside with the factors that facilitate and hinder this process. A qualitative methodology from the perspective of the interpretive paradigm was used, with a descriptiveexploratory methodological approach, the type of design that was used is symbolic interactionism, based on case study, where the sample is composed of three women with intellectual disabilities and the results were analyzed according to Grounded Theory.

The principal conclusion obtained in this investigation is that the meanings attributed by this group of women studied on having been woman and mother are the femininity and domestic tasks, joined emotional factors and of taken care basic. An important finding is that this group of persons is vulnerable to that there is a transgression of kind as the violation of his more basic human rights in his daily life, they do not exercise his significant occupations of independent form, being victims of a situation of Occupational Apartheid and Occupational Injustice.
\end{abstract}

\title{
Key words
}

Intellectual Disability, Women, Meaning, Maternity, Occupational Injustice, Human Rights. 


\section{INTRODUCCIÓN}

Actualmente la cifra de discapacidad en el mundo abarca el $10 \%$ de la población total, a nivel nacional la discapacidad corresponde a un $12,9 \%$, del cual, el $9 \%$ pertenece a la discapacidad intelectual, cifra que en la Región de Magallanes alcanza un 0,73\% de la población. Estas cifras demuestran que la discapacidad es un tema de importancia mundial, esta población cada vez toma mayor fuerza en diferentes ámbitos, especialmente en el derecho a ser madre, por lo tanto, la siguiente investigación nace a raíz de la inquietud acerca de los escasos antecedentes en relación a la temática de la maternidad en mujeres con discapacidad intelectual en Chile y en la ciudad de Punta Arenas.

Desde la visión de la Terapia Ocupacional es importante tomar en cuenta la ocupación de las personas, es por esto que la siguiente investigación analiza el significado de ser mujer y madre para el grupo estudio teniendo como antecedente la importancia que las mujeres le brindan al significado de la maternidad.

Existen diversas fuentes que sustentan esta investigación proveniente de organizaciones, leyes, estudios y autores tanto nacionales como internacionales, que son la base para el desarrollo de este estudio. Si bien no hablan directamente de la maternidad en mujeres con discapacidad intelectual, si hacen referencia a otras temáticas importantes, tales como género, discapacidad, maternidad, sexualidad, leyes, roles y la mirada desde la Terapia Ocupacional y Ciencia de la Ocupación.

El principal objetivo de esta investigación es analizar el significado de la ocupación de ser mujer y madre para un grupo de mujeres con discapacidad intelectual y cómo influye el contexto en el desempeño de las ocupaciones, además del ejercicio de los roles para el grupo estudio. Para lograr el objetivo principal se utilizó la metodología cualitativa según Taylor y Bogdan, (1987) desde un enfoque descriptivo exploratorio utilizando el interaccionismo simbólico para comprender el significado y la importancia de los roles que desempeñan estas mujeres como constructos sociales, centrándose en el estudio de caso, con base a la Teoría Fundamentada (Glaser y Strauss1967)

Desde la perspectiva de la Terapia Ocupacional es importante tener en cuenta que su base es la ocupación, la cual se caracteriza por el valor y significado que la persona le otorga a sus ocupaciones, por lo tanto, el enfoque de esta investigación, toma las ocupaciones de ser madre y mujer como actividades importantes dependiendo del significado que le brinde cada mujer a estas.

Para la Ciencia de la Ocupación el significado se entiende como:

"El significado de la ocupación se refiere a la experiencia subjetiva de la participación en ocupaciones. Las personas comunican a las ocupaciones el significado o el valor personal. Más aún las ocupaciones están simbólicamente constituidas en una cultura y son interpretadas en el contexto de las historias vitales de cada individuo". Crepeau, Cohn, Schell (2006) p. 17. 
Tal como se explica en la cita anterior los significados que se asignan a las ocupaciones son diferentes, por lo tanto para los hombres no tiene el mismo valor ser padre como para las mujeres el ser madre, ya que, viven un proceso diferente. Desde algo tan simple como ser padres surge una diferencia considerable en relación a la igualdad y equidad de género.

Las diferencias de género surgen debido a que cada cultura elabora una cosmovisión diferente sobre los géneros, es por esto que cada pueblo, cuidad, país y cada grupo de personas tiene un concepto de género basado en su propia cultura, partiendo de la base que cada pueblo tiene tradiciones nacionales populares, valores y una identidad cultural única.

Esta diferencia de género no solo se manifiesta entre la igualdad y equidad de género, también se ve reflejada entre las mujeres que poseen o no un tipo de discapacidad. Si bien la discapacidad puede ser innata o adquirida, transitoria o permanente, partimos de la base de que es un problema social (Horton y Leslie 1978), es decir, no exclusivo de la persona y su familia, sino de toda la comunidad. La característica principal del grupo estudio para esta investigación es la discapacidad intelectual, y sus múltiples desventajas en cuanto a temáticas como derechos reproductivos y maternidad que actualmente son ejes poco estudiados, pero de gran importancia para la población con discapacidad.

Ser madre tiene una significativa importancia para las mujeres, es una experiencia profunda y sobre todo íntima y personal que se va desarrollando a lo largo de la vida, pero es sabido que con la ayuda del núcleo familiar y los profesionales capacitados las mujeres con discapacidad logran aprender las habilidades necesarias para poder ejercer la maternidad y el cuidado de sus hijos sin mayor dificultad, así con el paso del tiempo lograr una independencia en la toma de decisiones con lo que respecta al cuidado y la crianza de los niños.

A partir de los antecedentes revisados, unido a la escasez de investigación en el país y en la comuna de Punta Arenas sobre el fenómeno de la maternidad en mujeres con discapacidad intelectual, surge la necesidad de investigar sobre los significados que tienen las mujeres con discapacidad intelectual con respecto a la experiencia de ser mujer y ser madre en la sociedad actual y a su vez, el impacto de estas vivencias en sus ocupaciones.

\section{MÉTODO}

En esta investigación se utiliza la metodología cualitativa, el paradigma al que se adscribe esta investigación es el paradigma interpretativo el cual puede ser estudiado objetivamente (Olabuénaga 2003). El enfoque de la metodología brindará una visión descriptivaexploratoria, ya que, pretende descubrir ideas, apreciaciones y datos desde nuevas perspectivas que amplíen las existentes, además de permitir conocer con mayor profundidad los relatos de vida en relación a la maternidad y la discapacidad intelectual dentro de su contexto sin intervenir en su ambiente natural, tomando en cuenta que tienen características propias y únicas al igual que todas las personas. Se conocerá desde la propia experiencia de las entrevistadas los significados como mujeres y madres con discapacidad 
intelectual, por último, la investigación se centra en el Estudio de Caso (Ottenberger, A. 2000).

Los lugares donde se observó la muestra son los domicilios de las mujeres.

El desarrollo de la investigación y la obtención de la información se recopilo a partir de entrevistas semiestructurada en profundidad.

Las participantes de la investigación fueron seleccionadas de acuerdo a los siguientes criterios de inclusión:

- Ser mujer

- Ser madre

- Poseer discapacidad intelectual diagnosticada.

- Ser mayor de 18 años

- Que resida en la ciudad de Punta Arenas

El proceso de análisis de datos recopilados en la investigación tuvo como directriz la Teoría Fundamentada.

En el desarrollo del estudio, se consideró e hizo valer los derechos de los participantes y sus familias. En este sentido, todas las personas entrevistadas y sus familiares directos ejercieron el derecho a aceptar voluntariamente a ser parte de la investigación. Firmaron un consentimiento informado documento a través del cual expresaron su voluntad de participar en la investigación manifestaron tener conocimiento absoluto sobre la investigación de haber sido informadas del propósito de la misma, así como de los objetivos y sobre uso de la información recolectada, exclusivamente para fines de la investigación. Además, se les aseguraba confidencialidad y anonimato, ejerciendo el derecho de no participar, de retirarse en cualquier momento o de negarse a contestar algún tipo de pregunta si lo consideraba oportuno (Rueda, 2002)

\section{RESULTADOS}

Los principales hallazgos que se obtuvieron en esta investigación es la falta de capacidad para crear un autoconcepto de ellas mismas como madres y mujeres, ellas más bien asocian a acciones concretas la elaboración de esta identidad debido a que poseen un coeficiente intelectual inferior al normal, por ejemplo, el significado de ser mujer lo asocian a la utilización de accesorios y el significado de ser madre se relaciona con el cuidado de sus hijos. (Peláez, 2009). 
En el siguiente cuadro se sintetiza la codificación selectiva del análisis de datos extraído de los significados que el grupo estudio atribuye a ser mujer y madre, en donde se expone que la cultura es quien influye y origina estos pensamientos en las mujeres.

En el esquema, se muestra la cultura como factor causante de los significados que las mujeres le atribuyen al ser madre y mujer y como esto influye en su nivel de dependencia y calidad de vida

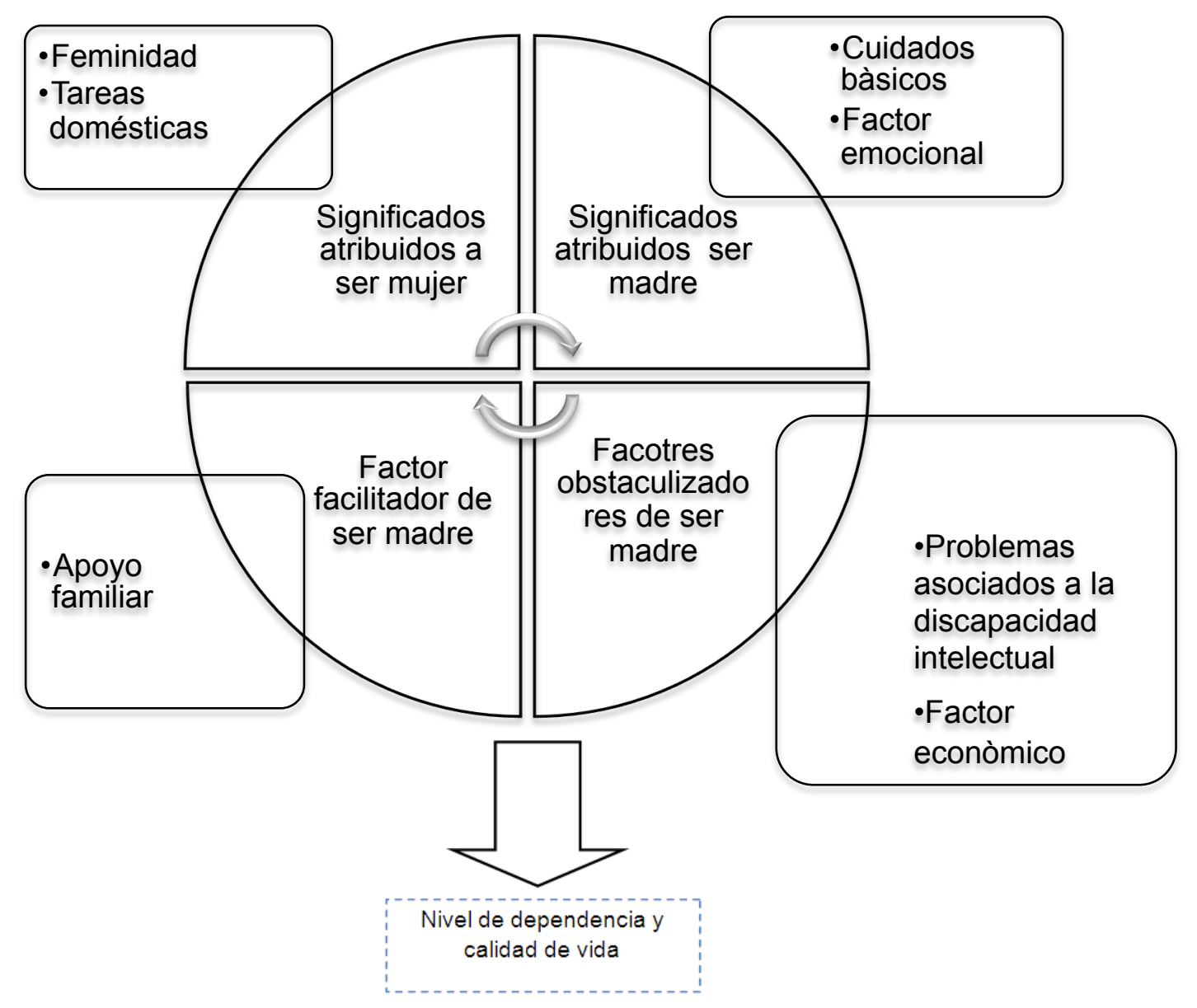

Algunos de los principales resultados son los siguientes

- Significados atribuidos a ser mujer: Desde la perspectiva de las entrevistadas, el ser mujer está asociado a la feminidad y a las labores domésticas como características propias del rol de ser mujer, las cuales están determinadas por la cultura y el contexto en el que se desenvuelven. Tomando en cuenta que estas mujeres tiene un coeficiente intelectual inferior al normal, demuestran la capacidad de reconocer su rol de una manera superficial, sin embargo, no perciben su identidad como mujer, esto se ve 
reflejado en los propios relatos de las entrevistadas quienes identifican que las mujeres deben ser femeninas aunque ellas no se sientan identificadas con esta cualidad, pero a lo que si le asignan mayor importancia es a la realización de las labores domésticas. Por lo tanto, se puede concluir que para este grupo de mujeres con discapacidad intelectual le atribuyen mayor significado a las labores domésticas que a la feminidad, debido a que el contexto y la cultura es un aspecto que influye en gran medida en su desarrollo personal.

- Significados atribuidos a ser madre: De acuerdo al relato de las entrevistadas, ellas identifican principalmente dos significados asociados. En primer lugar, el parte el factor emocional y por otro, los cuidados maternos brindados a sus hijos. Los sentimientos y emociones que sus hijos evocan en estas mujeres se ve reflejado en su discurso, ya que, la primera respuesta expresada fue el amor y cariño que sienten hacia sus hijos. Se pudo observar la presencia del instinto maternal, tanto en el significado del factor emocional, como en los cuidados maternos. Por ejemplo, el sentimiento de protección y la preocupación por el bienestar de sus hijos son elementos que nacen en cualquier madre sin importar su condición. En relación a los cuidados maternos estos fueron adquiridos en las mujeres estudiadas producto de una enseñanza por parte de la familia. La mayoría de los cuidados básicos que las mujeres estudiadas le brindan a sus hijos son supervisados por sus propias madres o por familiares cercanos para evitar que ocurra algún tipo de accidente.

- Factores facilitadores en relación a ser madre: De acuerdo al análisis realizado se pudo identificar que el apoyo familiar está presente en las tres mujeres entrevistadas siendo este un pilar fundamental para el desarrollo y desempeño de los roles de mujer y madre. La familia se preocupa de brindar toda la ayuda necesaria para que puedan desplegar sus habilidades de forma apropiada pero también se preocupa de que sean lo más independientes posible en su vida cotidiana junto a sus hijos. Una de las tres mujeres entrevistadas actualmente tiene pareja estable la cual cuenta con el apoyo económico, sin embargo, debido al trabajo que su pareja realiza no está presente la mayor parte del tiempo, las otras dos entrevistadas no cuentan con el apoyo del padre sus hijos, motivo por el cual sus hijos no cuentan con figuras paternales presentes.

- Factores obstaculizadores en relación al ser madre: A través del análisis realizado es posible identificar dos factores obstaculizadores importantes, por un lado la discapacidad intelectual y por otro el factor económico de este grupo de mujeres, aspectos que dificultan el desarrollo eficaz de la maternidad. El poseer discapacidad intelectual es un factor determinante que interfiere en varios aspectos en la crianza y los cuidados de sus hijos, ya que, al poseer un coeficiente intelectual inferior al normal acarrea la dificultad en reconocer ciertas convenciones sociales propias de la cultura en la que se ven inmersas, (Nuñez, 2007). de acuerdo a los factores económicos pudimos identificar que las mujeres entrevistadas tiene dificultades económicas, como se mencionó en el transcurso de la investigación, la pobreza y la discapacidad están 
íntimamente asociados. Además como poseen discapacidad intelectual tienen menos posibilidades de acceder a un trabajo remunerado estable, por el sólo hecho de poseer una discapacidad son marginadas y restringidas en el mundo laboral teniendo que depender en muchos casos sólo del dinero de sus familias, estos dos aspectos dificultan aún más la crianza de sus hijos.

\section{DISCUSIÓN}

Esta investigación espera establecer una base para las siguientes investigaciones en la línea de Salud Mental y Ciencia de la Ocupación en la región de Magallanes, abriendo una puerta inexplorada en el campo laboral donde los Terapeutas Ocupacionales logren un abordaje comunitario en la experiencia de la maternidad en mujeres con discapacidad intelectual bajo la mirada de los derechos humanos, analizando el significado de la maternidad como ocupación significativa para este grupo de mujeres.

Conjuntamente fomentar el desarrollo de políticas públicas, que aborden los factores obstaculizadores en relación a la maternidad en mujeres con discapacidad intelectual, (Dell'Anno 2004). por ejemplo: creación de programas específicos de salud destinados a las mujeres con discapacidad intelectual; en cuanto a la educación talleres sobre sexualidad y concepción, derechos y leyes, para eliminar la discriminación de género especialmente en las personas con discapacidad siendo uno de los grupos más vulnerados en la sociedad.

Mediante la realización y análisis de esta investigación se cuestionan los siguientes temas:

\section{Equipo multidisciplinario y atención primaria}

En base a las entrevistas realizadas y a los conocimientos adquiridos durante los años de estudio y el transcurso de esta investigación se identificó el diagnóstico errado por parte de los profesionales que se encargan de diagnosticar la discapacidad intelectual, ya que, en un principio la muestra debía abarcar a mujeres con discapacidad intelectual leve, al realizar la entrevista se pudo reconocer que a pesar de que las mujeres estuvieran diagnosticadas con discapacidad intelectual leve, poseían rasgos o características de una persona con discapacidad intelectual moderada o grave, además de diagnósticos asociados. Esto se debe a que los profesionales del área no realizan constantes reevaluaciones ni seguimientos a este grupo de mujeres, por lo tanto, se va generando diagnósticos errados que se mantienen en el tiempo, además de la carencia de un equipo multidisciplinario que trabaje en conjunto para la rehabilitación integral de estas mujeres y sus hijos.

Esta falencia debería ser tomada en cuenta por el sistema de salud, ya que, según la ley 20.422 de igualdad de oportunidades e inclusión social de personas con discapacidad, establece en el título III de prevención y rehabilitación lo siguiente: 
Artículo 20.

"Las medidas, planes y programas de prevención se adoptarán en consideración a los factores de riesgo de discapacidad, en especial, enfermedades agudas y crónicas, lesiones, accidentes viales, laborales y de cualquier otro tipo, violencia, problemas de calidad ambiental, sedentarismo, abuso del alcohol o las drogas, tabaquismo, desórdenes nutricionales, maltrato infantil, condiciones sanitarias deficientes o estrés."

Si bien este grupo de mujeres cuenta con el apoyo de su familia, no recibe la ayuda por parte del sistema de salud, ya que, este grupo de mujeres con discapacidad no cuenta con talleres ni programas de apoyo específicos en todo el proceso pre, peri y postnatal, de sus hijos, donde abarquen temas como: educación sexual, esterilización, métodos anticonceptivos, concepción, paternidad responsable, estilos de crianza, estimulación temprana, entre otros.

\section{Violación de los Derechos humanos}

Según los antecedentes revisados en este estudio él ser madre, mujer y además poseer una discapacidad intelectual no deberían ser causa de discriminación, pero actualmente esta población es la más vulnerada de la sociedad violando sus derechos más básicos (Eroles, 2005). El primer lugar el rol de ser madre es un derecho que se ve intervenido por la familia, los profesionales, el contexto y en general por la sociedad, ya que, por distintos motivos estas personas suelen tener mayor grado de responsabilidad sobre sus propios hijos que ellas mismas, quebrantando su derecho a la maternidad y segundo lugar el solo hecho de ser mujer y poseer discapacidad intelectual las pone en un lugar más desventajoso con respecto a las otras mujeres teniendo menos oportunidades de acceso a realizar actividades y ocupaciones. Situación que toma en cuenta la Convención de los derechos de las personas con discapacidad, que ha promulgado nomas con respecto a este tema, pero que no están siendo cumplidas en su totalidad, a pesar de que Chile es un estado parte. En el Artículo 16 Protección contra la explotación la violencia y el abuso se puede reflejar lo siguiente:

"Los estados partes adoptaran todas las medidas de carácter legislativo, administrativos, social, educativo y de otra índole que sean pertinentes para proteger a las personas con discapacidad, tanto en el ceno del hogar como fuera del él, contra todas las formas de exploración, violencia y abuso, incluidos los aspectos relacionados con el género". ONU, (2006), P.13

Es importante mencionar que a pesar de todos los avances que ha habido en todos estos años en relación a la discapacidad aún sigue siendo un tema que se oculta, queda reflejado en estas mujeres quienes son restringidas a vivir en sus hogares limitando sus posibilidades ocupacionales. 


\section{Injusticia ocupacional}

Desde la visión de la Terapia Ocupacional estas mujeres con discapacidad intelectual que son madres están viviendo una situación de injusticia ocupacional, ya que, en comparación con otras personas tienen limitadas su participación en distintas ocupaciones, debido a la situación geográfica de la región, el perfil de doble discriminación, a la violación de sus derechos y en general cualquier factor o variable que influya en la restricción del acceso a ocupaciones va a desencadenar en una situación de injusticia ocupacional.

Según el capítulo "Justicia Ocupacional" de Wilcock y Townsend en el libro Willard \& Spackman Terapia Ocupacional la justicia ocupacional se entiende como: "La aplicación al derecho de todo individuo de poder satisface las necesidades básicas, y tener oportunidades y posibilidades en la vida que sean equitativas para alcanzar su potencial, pero específico a su participación en ocupaciones diversas y significativas". Wilcock, Townsend, (2011), p.193

\section{Apartheid Ocupacional}

El que las mujeres con discapacidad posean esta condición es una característica que es innata la cual permanecerá de por vida, haciendo que se encuentren segregadas de sus ocupaciones más significativas al cumplir el rol de mujer y madre. Otro factor importante es el nivel socio económico al que pertenecen las entrevistadas, factor que restringe la oportunidad de participar en ocupaciones significativas a las mujeres con discapacidad intelectual, desempeñando su rol de mujer y madre en la sociedad actual. Por ultimo, el género también provoca que las mujeres con discapacidad intelectual no tengan igualdad de oportunidades en la participación de ocupaciones dignas, solo por el hecho de ser mujeres.

Desde la visión del libro Terapia Ocupacional sin fronteras, Aprendiendo del espíritu de supervivientes en el capitulo VI superar el apartheid ocupacional, exploración preliminar de la naturaleza política de la terapia ocupacional define apartheid ocupacional como:

“La segregación de grupos de personas mediante la restricción o negación de su acceso a una participación digna y significativa en las ocupaciones de la vida diaria, basada en la raza, color, discapacidad, procedencia nacional, edad, sexo, orientación sexual, religión, creencias políticas, estatus en la sociedad u otras características. Ocasionando por fuerzas políticas, sus consecuencias sociales, culturales y económicas, sistemáticas y dominantes, ponen en peligro la salud y el bienestar de individuos, comunidades y sociedades." Kronemberg, Pollard (2007), P. 66 


\section{Transgresión de género}

Hoy en día a pesar de todos los avances que ha habido en la sociedad, sigue existiendo la violación o transgresión de derechos, en este caso este grupo de mujeres la gran mayoría de las veces son alejadas de la posibilidad de poder expresarse y demostrar libremente su opinión o lo que sienten, pasando por una situación de invisibilidad social ante la población, esto ocurre por que son ocultadas y renegadas ante la sociedad, por el solo hecho de ser mujer sumado a que poseen una discapacidad, por lo que esta problemática muchas veces se vuelve un tema tabú en nuestra sociedad, es algo que sucede a menudo, sin embargo, se mantiene en un bajo perfil.

Por otro lado, la misma sociedad hace que halla menos oportunidades para estas mujeres con discapacidad que para los hombres con discapacidad, por ejemplo, brindando más oportunidades laborales a estos con una mejor remuneración.

La transgresión de género en las mujeres con discapacidad es un tema que se esta desarrollando cada vez mas, es así como Pilar Gonzales en su estudio: Las mujeres con discapacidad y sus múltiples desigualdades; un colectivo todavía invisibilizado en los estados latinoamericanos y en las agencias de cooperación internacional, hace referencia a:

"El género y discapacidad interactúan colocando a las mujeres con diversidad funcional (discapacidad) en una posición desigual respecto a los hombres y a las personas sin discapacidad. Por lo tanto, ellas sufren un mayor índice de marginación y exclusión social que desemboca en una violación de los derechos humanos más básicos." Gonzalez Rams, (2010) p.6

\section{Sugerencias:}

A partir de ésta investigación surgen las siguientes sugerencias para mejorar la calidad de vida de estas madres y de sus hijos.

- Es importante como Terapeutas Ocupacionales participar en la colaboración diagnostica, para evitar diagnósticos errados y así poder identificar habilidades que presenta la persona y ayudar a que desarrolle al máximo sus capacidades, también es importante crear conciencia en el equipo multidisciplinario para realizar evaluaciones constantes y un seguimiento continuo especialmente en estas mujeres y sus hijos, llevando un registro de todos los casos de mujeres con discapacidad intelectual que existen en el centro de salud familiar u hospitales, para poder crear planes y servicios específicos dirigidos a ellos y a sus necesidades más importantes. La importancia de contar con estadísticas permite .que los países y las instituciones puedan planificar y focalizar de mejor manera las acciones tendientes a contribuir a la inclusión social de las personas con discapacidad. El equipo multidisciplinario que 
trabaje con este grupo de mujeres es importante que el tratamiento se aborde desde un tratamiento integral tanto para la madre como para el hijo para asegurar que estén cubiertas todas sus necesidades, esta atención es trascendental que sea de forma prolongada para lograr cambios importantes en la vida de las mujeres con discapacidad.

- Otra sugerencia importante es la realización de talleres en los centros de atención primaria de salud, centros de salud familiar u hospitales, estos talleres deben estar dirigidos a las madres con discapacidad donde se pueden desarrollar temas como sexualidad, esterilización, métodos anticonceptivos, concepción, paternidad responsable, estilos de crianza, para que adquieran destrezas o habilidades en relación al cuidado y crianza de sus hijos, además del aprendizaje que ellas mismas tendrán sobre su cuerpo, por ejemplo, el saber como amantar a su hijo; a su vez talleres destinados a los menores como por ejemplo de estimulación temprana y estimulación sensorial.

- Teniendo una base teórica que sustenta nuestra investigación en donde además se hicieron hallazgos que interfieren negativamente en este grupo de mujeres, se puede llevar a cabo un proyecto a mediano o largo plazo dirigido a los centros de atención primaria, hospitales u otro centro que favorezca a este grupo de mujeres con discapacidad y sus hijos, con la realización de este proyecto se abrirán nuevas puertas en el ámbito laboral para futuros Terapeutas Ocupacionales.

- Con respecto a las investigaciones futuras que se realicen en torno a esta temática se sugiere que al momento de identificar al grupo estudio se corrobore el diagnostico para asegurar que sea el adecuado, además se propone la realización de entrevistas al núcleo familiar para conocer en detalle la visión que tienen ellos de acuerdo a la maternidad de estas. También se podría abordar el estudio desde otra perspectiva haciendo un estudio comparativo en relación a los significados atribuidos a ser mujer y madre entre las mujeres con y sin discapacidad.

\section{REFERENCIAS BIBLIOGRÁFICAS}

AOTA. (2008). Marco de trabajo para la práctica de Terapia Ocupacional: Dominio y Proceso. .

Crepeau, C. S. (2006). Willard \& Spackman Terapia Ocupacional. Buenos Aires: Editorial Médica Panamericana.

Dell'Anno,A. (2004). Alternativas de la diversidad social: Las Personas con Discapacidad. Buenos Aires: Editorial Espacio.

ENDISC. (2004). Primer estudio nacional de la discapacidad. Chile. 
Eroles, C. (2005). La Discapacidad: una cuestión de Derechos Humanos. Buenos Aires: Editorial Espacio.

Glaser, B. Strauss A. El descubrimiento de la Teoría Fundamentada. Estrategias para la investigación cualitativa. Sociología Press. 1967

González Rams, P (2010). Las Mujeres con discapacidad y sus múltiples desigualdades; un colectivo todavía invisibilizado en los Estados latinoamericanos y en las agencias de cooperación internacional. p. 2737-2756 [ $14^{\circ}$ Encuentro de Latinoamericanistas Españoles (. Santiago de Compostela)]

Horton, P. Leslie G. “Problemas sociales”. Ed. El Ateneo. Buenos Aires. 1978

Kielhofner, G. (2002). Modelo de Ocupación Humana, Teoría y Aplicación. USA: Panamericana.

Kronenberg, S. A. (2007). Terapia Ocupacional sin Fronteras: Aprendiendo del espirítu de supervivientes. Buenos Aires, Bogota, Caracas, Madrid, Sao Paulo: Editorial Medica Panamericana.

Nuñez, B. (2007). Familia y discapacidad: De la vida cotidiana a la teoría. Buenos Aires: Editorial Lugar.

Olabuénaga, J. I. (2003). Metodología de la investigación cualitativa. España: Universidad de Deusto Bilbao.

ONU. (1993). Normas uniformes sobre la igualdad de oportunidades para las personas con discapacidad de las Naciones Unidas.

ONU. (2006). Convención sobre los derechos de las personas con discapacidad de las Naciones Unidas.

Ottenberger, A. (2000). El estudio de casos en la Investigación Social. Santiago de Chile: Universidad Tecnológica Metropolitana.

Peláez, A. (2009). Maternidad y Discapacidad. España: Editorial Cinca.

Rueda, L. (2002). Principales dilemas bioeticos en las personas con discapacidad prolongada. Chile. Revista Chilena de Terapia Ocupacional. Santiago.

Taylor, S. Bogda R.. (1987). Introducción a los métodos cualitativos de investigación. Barcelona, Buenos Aires, México.: Editorial Paidós.

Willard, H. Spackman; C. Blesedell Crepeau; E. Cohn; E. Boyt Schell, B. Madrid; Buenos Aires. Médica Panamericana. 2010 